\title{
EDUCATION AND TECHNOLOGY DURING COVID-19 TIMES
}

\author{
Daniel Nicolas Dominguez-Perez ${ }^{1}, \&$ Nicolas Dominguez-Vergara ${ }^{2}$ \\ ${ }^{1}$ Universidad Nacional Autónoma de México (Mexico) \\ ${ }^{2}$ Universidad Autónoma Metropolitana (Mexico)
}

\begin{abstract}
It is difficult to separate technology from education because technology is embedded in teaching. The best technology for teaching is the one that does not interfere with the communication between the teacher and the students. In other words, it should be barely "noticeable" in how it is used, and easy enough to use that it does not require special training, such as window in a room to see the flowers outside, without interfering in any way. The technology used during an in-person classes is so basic, transparent, and simple, since it consists of a classroom, chairs, blackboard and pieces of chalk. However, a piece of chalk could break. In this case, the flow of information is interrupted; which is why the plastic whiteboards and markers are preferable and cleaner. An electronic board may seem to be better with its many improvements with computers; however, its higher technology could get in the way if it is not used properly. During the pandemic, different technologies, like computers and cell phones, connected to internet were massively used. Yet, computers and cell phones with the appropriate software and apps had an additional cost; with no guarantee of antivirus applications against viruses or worms, leaving aside protection for documents, software and hardware. On top of that, a Zoom, Google or any similar online platform was necessary. Using these platforms, other technologies had to be relied on, including Ethernet or a USB adapter. On the other hand, in developing countries before the pandemic, low-tech was used by most students to store and pass information to their peers and teachers. They would use mainly pens and notebooks to write notes, or CD-Rs or USB to pass information. However, during the pandemic, with the advances in internet speed, many students have now moved on to using e-mail and cloud drives where they can transport their files. All the electronics listed to make online education or tech-based education needed electricity to work. In other words: no electricity, no class; no electric supply or stored electricity in batteries, no class. In this paper we analyze the role of technology in education, and how this technology could enhance or obstruct the communication between the teacher and the students.
\end{abstract}

Keywords: Education, technology, COVID-19.

\section{Introduction}

Before the COVID-19 pandemic most of the students all around the world used paper notebooks and pens or pencils to take notes in class; mainly in public universities and developing countries. The students in class had a direct contact with the teachers and all of them, once inside a classroom, were isolated from the outside world. The communication was transparent as a crystal window to see the flowers outside. There was no interference of any electronic technology between the students and the teacher; if the teacher asked a question the students responded on the spot. The teacher never had to ask, "are you there?", “do you hear me?", “do you see what I am writing?" The classroom was a safe haven for teachers and students; there were less distractions and less exposure to outside dangers, with no family members asking questions or children interrupting. In case of the need of internet, the signal was usually present, provided by the school. After class, the students would close their notebooks, where they would store their information from class. If the notebooks got wet, the information, the physical information, was lost forever; as well as if the notebooks were stolen or lost. And then, suddenly, the COVID-19 pandemic came dismantling the educational systems of many countries of the world. Countries rushed to teach online; that is, using computers for the communication between teachers and the students. These computers could be anything from smartphones to tablets, laptops, or desktops. In some parts of underdeveloped countries, computers were not affordable by all students and teachers. When the internet signal was too expensive to reach, of bad quality, or perhaps non-exist, the elementary and secondary education was held through digital television or even through radio. Online education is based solely 
upon technology. If the technology fails or the electricity fails, there is no communication and consequently, no class. The link to communicate both the teachers' and students' computers, is the internet. Along with the computers and the link between them, there are other technologies used to facilitate the interaction between the teachers and the students: Zoom, Google Meet, along with other online platforms such as WhatsApp, electronic mail, and others. In this paper, the role of technology in education is discussed and some thoughts are given to the future of education.

\section{Technology and education}

Technology is a tool which helps carry out an activity. It could be hard and soft. Hard technologies are those which are physical tools that aid in completing tasks. Examples of these can range from simple cups to drink water, to stoves to cook and prepare food, to vaccines, to computers, to weapons, and even to space probes. Soft technologies differ in being tools that are either intangible or imaginary that would require a medium for it to be used. Examples of soft technologies are procedures (recipes) to fabricate cakes or series of instructions to be interpreted by machines such as software. Viewed in this way, it is the computer software used to write a text in a virtual document in a computer. Many of the technologies we know are the result of inventions and innovations since the first human appeared on earth. The technology can be low, medium or high. High technology is invented or innovated using advanced knowledge of physics, chemistry, mathematics or other sciences. Examples of high technology are vaccines against COVID-19, laptops, wireless interfaces, and computer software. On the contrary, low technology does not require the most advanced knowledge to be created, for example: notebooks, pens, pencils or blackboards. Developed countries dominate the inventions and innovations of high technology. Differently, underdeveloped countries acquire some high technology through purchase in the world market, because it is sold in order to recover the investment for its development, with exception to technologies that may jeopardize safety or security such as advanced weaponry or newly invented encryption algorithms. Underdeveloped countries do not spend money in developing high technology. This is why they are at the expense of what developed countries create, which will not be necessarily appropriate for their needs.

Education is the collection of training and exercises used to prepare a person enough to independently perform tasks in their professional and daily life. All people receive some form of education whether it be institutional or by the community around them. Most people who do not receive a formal education will learn something from their own families and communities. A formal education through schooling is important to be able to be part of the larger world community that exists beyond the family or local community. Currently, it is often believed that the necessity of an education is for people to be able to become qualified for working or for starting a business. While receiving an education will allow a person to reach better opportunities it is not the only reason an education is important. A proper education is necessary for people to know and understand the world and society around them while also having a proper understanding on the events that may be happening in the world.

Education could be also defined as the systematic process to transmit knowledge and methodologies to acquire abilities from a teaching agent to a student through the communication between them. The teaching agent before the pandemic in the developing countries was mostly the teacher, in flesh and blood, who met the students in a convened physical place to communicate. This communication was mainly through voice, eyes, physical language, and handwriting on the blackboard or whiteboard with chalk or markers, respectively. In this sense, the technology used for education prior to the pandemic was very basic: a classroom in a building, chairs, blackboard, whiteboard, chalk, markers, notebooks, and subject books. The teaching of some knowledge and abilities requires special equipment like computers, chemical equipment, medical specialized devices, and even well-equipped laboratories and workshops (to feel, to test, and to practice). Occasionally, the location for in-person education could temporarily move to other places, such as hospitals in the case of medical careers, or to factories in the case of industrial engineers. During the pandemic the teaching agent was not physical, also, there was not a physical place to meet, and the communication was through computers and internet. Technology for education consists of all of the tools that are involved in allowing the teacher to communicate with their students and helping them understand the subjects of the courses. This includes markers and dry-erase boards for the professor to communicate with their students, besides the writing tools that students use to capture the information and to create notes, such as notebooks, paper, pencils, pens, or in some cases, computers and tablets with their respective software used for note taking.

Now, the teaching-learning process takes place at home; the computers are not in the physical school, nor is the teacher-student communication. Actually, the school is distributed in all of the students' computers and the teacher's computers. As online education is technology-based and this technology has its cost, a lot of teachers and students must pay for the new tools of the learning-teaching process. 
Financing in many cases the schools they work for or study in; similar to Uber and Rappi workers, who use their own vehicles to work for their companies. Therefore, the families all around the world had extra expenses in buying the technologies to teach or to learn. In Mexico, the percentages of dwellings with telephone for members in the range of 3 to 29 years old spent additional money to attend online classes due to COVID-19. Analyzed by type of expenses: smartphone, 28.6\%; fixed internet service, $26.4 \%$; chair, table, or other furniture to condition space to study, 20.9\%; laptop or desktop, $14.3 \%$; other electronic devices, $6.8 \%$; prepaid phone plan, $6.2 \%$; digital television, 5.2\%, and tablets, 5.1\% (INEGI, 2021). The advantages of online education according to the population of 3 to 29 years old are (INEGI, 2021): limited health risks for students (56.4\%); allows family life (22.3\%); savings in transportation and school materials, $(19.4 \%)$; no time loss in transportation $(15.0 \%)$, and fosters self-learning and self-study abilities $(8.5 \%)$. Interestingly, there were those who did not notice any advantages $(12.4 \%)$. The disadvantages according to the same survey are: there is no learning or little learning (58.3\%); there is no follow-up on the learning of the students $(27.1 \%)$; lack of pedagogical technical capacity or ability of parents/tutors to transmit knowledge to students (23.9\%); excessive homework and scholar activities $(18.8 \%)$; no appropriate space at home $(18.2 \%)$ and lack of coexistence with friends and classmates $(16.1 \%)$.

\section{Physical classrooms versus virtual tools}

The teacher needs to communicate with various students at once in a classroom or a group. To do this, it is necessary to have a medium that allows every student to view the information presented by the teacher. For many years, a blackboard has allowed this by providing a large space to write and draw, with enough clarity for students to read properly. The blackboard has been used for many years to provide this. The technology used here is transparent enough to allow this communication to be almost seamless, until a piece of chalk breaks or when it is time to erase the board. The whiteboard has become the replacement for chalkboards, which has allowed a much more clean and seamless transition between subjects, and facilitating the continuity of the class due to the use of dry-erase markers, which are much easier and faster to erase. Primarily, there is very little need to learn on how to switch from a chalkboard to a whiteboard. It is however, necessary to know that a specific type of marker must be used on these boards to prevent permanent or semi-permanent marks, or to prevent smudges on the board that will need to be cleaned later. Various companies in computation and digital interfaces have attempted to create a new replacement for the whiteboard by integrating computer technology, but it is still far from completely replacing the traditional whiteboard. In the adaption of computer technologies, many professors have adopted the use of projectors which has allowed a better visual communication with the students in the class. However, in many cases these classes are mostly given in a digital presentation that are either not well prepared enough or they only use the minimal number of tools that the presentation software has to offer. When a classroom includes the use of a computer, such as a desktop or a laptop and a projector, the teacher is able to prepare the class more easily and quickly, as the previously prepared material can be shown instantly before the students. However, there are moments in which this communication is either delayed or interrupted when there are problems such as software errors, hardware errors, power outages, or a limited ability in the use of the tools. All of these cause the computer and projector to lose their transparency as teaching technologies in the classroom. There may also be cases in which the software itself has features that are more of a distraction than helpful tools, such as attempts of software companies that add unnecessary features in virtual whiteboard software or other programs that may be used for teaching. Virtual education has become a necessity during the pandemic as a way to avoid physical contact and possible infection between students by moving all classes at home. Due to this, professors and students needed to adapt to classes that were in a similar style to the classes given either through presentations or through a webcam, with the professor explaining in the closest manner they could to using a whiteboard. During the pandemic, platforms were used where students could consult the course plan, classes, assignments, projects they have to carry out, and to also "turn in" their homework, exams and assignments; the google classroom is one of them.

\section{Notebooks, USB, and storing in the cloud}

When students take notes and work on assignments, they also need a way to write and store them. In most cases this is done through handwritten mediums using notebooks, binders, or loose paper. Prior to the pandemic, most teachers would hand out photocopies of worksheets or questionnaires for the students to complete and turn in to the class. It is very common for students to write reports and essays on a computer as it is more readable and cleaner than handwritten tasks. It should be noted that these reports would commonly need to be printed and turned in physically to the teacher. Due to this, schools were 
obliged to provide computers and printers in their campuses for students to complete their work for those who did not have easy access to a computer away from school. It was however necessary to have a method of portable storage for the students to carry with themselves, and use for editing and printing documents when needed. There have been also cases in which students no longer had to print and instead, turn in their assignments digitally. It was very common in earlier years for assignments, such as multimedia projects or programming assignments, to be turned in on floppy disks and writable CD's. When USB flash drives appeared they became more popular due to having a higher storage capacity and were easier to manage files. These have been used more for holding multiple files for the student to carry with themselves to be able to either work on different computers in the computer labs of the school, or to take the file to a printer. As teachers became more familiar with internet technologies, such as email, they would begin to simply ask their students to send their completed assignments to them directly, at any time, before a deadline. In other cases, students have been able to use email and cloud storage to replace the use of their USB drives where it is even possible to access the document they are working on and edit it from their cell phones.

\section{The future of education and technology. The end of physical teachers?}

The teaching agent in the future may not be physical for a considerable number of careers, in which software and apps could store all the information of courses, including information with sound and video that may help transmit the information necessary to the students. More advanced software might be constructed, such as one that could answer any student's questions (by uploading to the computer's site a great deal of possible questions beforehand, choosing the closest to the question asked by the student, and answering with the recorded answer). All this can be possible in the future and, in the long term, improved with artificial intelligence, internet of things, augmented reality, and other technologies. This could be the end or the replacement of physical teachers by virtual teachers in the future; although not completely. In that manner, teachers in Modern schools could be in jeopardy at least at certain degree; but not in the underdeveloped countries, for instance Mexico, where only $40 \%$ of the households can afford to buy a computer. In the developing countries for many economic activities, the labor force is still cheaper compared to the investment of acquiring and using robots (Dominguez-Perez et al., 2017) that is partly why automation has not greatly advanced. What is taught in education is knowledge that is complex to understand or abilities that are not easy to acquire (Bobbitt, 1915). Therefore, there is the need of a teaching agent to help understand the concepts or to help to acquire the mental or physical abilities required. The knowledge and abilities needed in the world will change in the future. Accordingly, the careers will change, the specializations may flourish, and, therefore, the virtual teachers would be prepared with the information to form special professionals. If this scenario materializes, the developing world will be at the mercy of developed countries because the latter will have the technology to produce the virtual teachers and the courses. If this is the case, the underdeveloped countries will have to define strategies for their academic independence from the advanced countries, since the developed technology will be appropriate for the culture, race, economy, habits, religion, songs, and society's needs of the same countries that have developed the technology. For example, some technologies are biased to work effectively to only a limited amount of people because the developers only took into consideration the characteristics of the people they know locally.

Being the communication crucial for education, it is clear that previsions have to be taken in the future for other types of communications. For example, sign language could be convenient to be used in certain situations, such as removing the language barrier that currently exists between deaf and hearing people, maintaining communication with a person who has lost their ability to hear or even to be used in situations where spoken communication is not viable such as noisy environments or situations where silence is necessary. It is unknown what tools, including those of communication, will be more crucial during future climate change disasters. It is still unknown what could specifically happen due to climate change yet, but it is important to make as many predictions as possible. If the world continues developing the information and communication technologies, their use will become even more massive in the future and, therefore, it is time to start developing strategies to assure their future production in distributed places, and not just the production that is convenient for certain places. For example, during the progression of the COVID-19 crisis there is a shortage of microchips, and therefore the massive production of electronic devices which are needed in several economic sectors is compromised. Consequently, the dependence of microchips should be addressed for future crises. Likewise, the dependence on electricity in order to assure alternate and direct distributed electricity all around the world. Also, affordable technology to store electricity should be developed, because laptops, cell phones, and other technologies depend on batteries. Electricity should be obtained from clean renewable sources, 
and hydrogen technologies should develop in order to use them to safely produce affordable and clean electricity.

In the future, in developed and rich countries, there will be in-person, online and hybrid education. However, in poor countries in which education is secular, free and obligatory, like in Mexico, most schools will only offer in-person education because online education requires a lot of technology, which will not be affordable for all students, as it is being documented during the COVID-19 pandemic. The surviving private schools will make cost-benefit studies to decide what mix of in-person, online, and hybrid education will offer, along with which careers to teach. Online education will require less infrastructure and less physical teachers, but there are families with barely enough economic resources to have their children educated in in-person schools, no matter how expensive they might be; and in those schools, physical teachers will do the teaching.

In the past, there were short technical careers taught by sending the course material by mail. It is possible that in the future, the whole course material, including all homework and tests, could be in internet sites that students can download just one time to work with at home. These will require no internet, because students would have stored all the information in their computers. In this type of education, the students could upload the answers of their homework and tests to the school's internet sites once finished. In this type of education, there might not be a school calendar and the students could start at any time a course or a career. There may be several educational systems that could be created in the future; it would not be solely in-person, online, and hybrid education. Technology is important for countries to advance forward, and gain advantages in areas such as economy and defense. As such, it is noted that developed countries will have high competition in these areas. Countries with more advanced technologies of weaponry have conquered, or are still conquering by force other countries' land. Although not only powerful technology is sufficient to defeat adversaries or enemies, but also the strategies used. There are plenty of examples of this in the many wars around the world in the history of humanity. So, it is possible that from now on, the technology for education can be used to quickly prepare technicians or professionals in certain strategic fields of science and technology to create a valuable edge to overcome competitors. For example, the competition to conquer the world market between the United States and China is already happening: China is the largest producer of electric cars while the United States has fallen behind. The United States has lost expertise and know-how in the manufacturing of several critical technologies and requires new educational policies to have young educated people who can become the top-notch leaders in the world of science and technology. This would not be easy, given the fact that higher education in the United States is very expensive. Online education has increased the socioeconomic inequality in the world; the disparity in the future involving online education among the countries in the world will be an indicator of the further inequality and poverty, which will be generated. Online education is not exclusively to learn skills and knowledge but to understand, manage and dominate other ways to communicate, and socialize. It involves mastering new technologies, which can make the students acquire advantages for getting better jobs.

\section{Conclusions}

The COVID-19 pandemic uncovered the challenge of educating the world through computers and internet, technologies which are not as transparent as whiteboards and markers. The crisis has opened the possibilities of new systems of education, which could trigger further socioeconomic inequality in the world and even inside a country, region or city. The world should anticipate the technology and resources needed for future crises based on the experience of the one that is, apparently, ending.

\section{References}

Bobbitt, J.F. (1915). The Project Gutenberg eBook, What the Schools Teach and Might Teach. Retrieved 23 May, 2021, from: https://www.gutenberg.org/ebooks./13482

Dominguez-Perez, D.N., Dominguez-Vergara, N., Dominguez-Perez, R.M., \& Pantoja-Gallegos, J.L. (2017). A future of isolation: control and automation in the developing countries. ICERI 2017 Proceedings. pp. 7964-7973.

INEGI. Encuesta para la Medición del Impacto COVID-19 en la Educación (ECOVID-ED) 2020. (2021). Retrieved 23 May, 2021, from: https://www.inegi.org.mx/contenidos/investigacion/ ecovided/2020/doc/ecovid_ed_2020_nota_tecnica.pdf 